\title{
Quantitative analysis of soft-bottom molluscs in the Bellingshausen Sea and around Peter I Island
}

\author{
Jesús S. Troncoso, ${ }^{1}$ Cristian Aldea, ${ }^{2}$ Patrick Arnaud, ${ }^{3}$ Ana Ramos ${ }^{4}$ \& Francisco García ${ }^{5}$ \\ 1 Departamento de Ecología y Biología Animal, Facultad de Ciencias del Mar, Campus Lagoas Marcosende, 36310, Universidad de Vigo, Spain \\ 2 Departamento de Ecología y Biología Animal, Universidad de Vigo, Spain, and Fundación Centro de Estudios del Cuaternario de Fuego-Patagonia y \\ Antártica (CEQUA), Avenida Bulnes 01890 - Casilla 113-D, Punta Arenas, Chile \\ 3 Le Jas des Batarins, 04110, Vachères, France \\ 4 IEO Centro Oceanográfico de Vigo, Cabo Estai, Vigo, Spain \\ 5 Departamento de Sistema Fisicos, Qvimicos y Naturales, Facultad de Ciencias Experimentales, Universidad Pablo de Olavide, Sevilla, Spain.
}

\section{Keywords}

Soft-bottom; Molluscs; Bellingshausen Sea; Peter I Island.

\section{Correspondence}

Jesús S. Troncoso, Departamento de Ecología

y Biología Animal, Facultad de Ciencias del

Mar, Campus Lagoas Marcosende, 36310,

Universidad de Vigo, Spain.

E-mail: troncoso@uvigo.es.

doi:10.1111/j.1751-8369.2007.00033.x

\begin{abstract}
Macrobenthic soft-bottom molluscs were sampled in 30 stations located in the Bellingshausen Sea at depths ranging from 90 to $3304 \mathrm{~m}$. The samples were collected using a quantitative grab box-corer during the cruises BENTART 03, from 24 January to 3 March 2003, and BENTART 06, from 2 January to 16 February 2006. Molluscs represent 1074 specimens belonging to 62 species of Polyplacophora, Gastropoda, Bivalvia and Scaphopoda. The bivalve Cyamiocardium denticulatum was the most abundant species (448 specimens). The abundance per station varied between 1 and 446 specimens. The Shannon-Wiener diversity index ranged between one specimen and 2.36, the Pielou evenness index ranged between 0.00 and 1 and species richness ranged from 1 to 14 species. Diversity showed great variations at different stations. After multivariate analysis (cluster analysis and nonmetrical multidimensional scaling) based on Bray-Curtis similarities, we were able to separate two principal clusters. The first cluster groups together species from shallower bottoms near Peter I Island and the Antarctic Peninsula, and the second cluster groups together species from deeper bottoms in the Bellingshausen Sea. The combination of environmental variables with the highest correlations with faunistic data was that of depth and coarse sand at the surface.
\end{abstract}

Information about the benthic macrofauna of the Bellingshausen Sea and Peter I Øy (Peter I Island) was scarce. To date, approximately 895 species of gastropods and 379 species of bivalves are known from the Southern Ocean and adjacent regions (Linse et al. 2006); Clarke et al. (2004) mentioned approximately 530 species of gastropods and 110 of bivalves found exclusively in the Southern Ocean. Only three families and 11 species were recorded in the Bellingshausen Sea, and two families and three species were recorded at Peter I Øy. This apparently low number of species is the result of poor sampling of this area: two samples on the continental shelf (1$1000 \mathrm{~m})$, none on the continental slope (1000-3000 m) and six deeper $(>3000 \mathrm{~m}$ ) have been obtained in the Bellingshausen and Amundsen seas out of 1490, 98 and
36 respective samples performed in these three zones in the whole Southern Ocean (Clarke et al. 2004).

The BENTART 03 (from 24 January to 3 March 2003) and BENTART 06 (from 2 January to 17 February 2006) research programs were carried out on board the RV Hesperides in the Bellingshausen Sea and off Peter I Øy. Benthic molluscs have a particularly wide ecological and ethological spectrum. So the assessment and analysis of their assemblages is likely to contribute to a better understanding of the structure and interactions inside the more complex benthic assemblages in which they live and interact.

As an integrated study of the benthic ecosystem, the BENTART programme is a good opportunity for analysing malacological assemblages. On the basis of the data obtained during this survey, we try to answer the 
following questions. Is the molluscan fauna really poor in the Bellingshausen Sea and nearby areas? How many molluscan assemblages are living there? What are their species diversity and dominant species? What are their ecological characteristics or requirements?

\section{Material and methods}

\section{Study area}

During the research cruises BENTART 03 (JanuaryFebruary 2003) and BENTART 06 (January-February 2006) molluscs were collected, together with other benthic invertebrates, at 40 stations in the Bellingshausen Sea, from the Antarctic Peninsula to Thurston Island on the border of the Amundsen Sea (Fig. 1), at depths ranging from 90 to $3304 \mathrm{~m}$ (Table 1). Because of the weather conditions (big waves, heavy winds) the box-corer cannot work in all sampling stations, and for this reason only 30 stations were sampled and used in the present quantitative studies.

\section{Sample collection}

In each station, the samples were collected using a quantitative grab box-corer (BC) with a maximum break- through of $60 \mathrm{~cm}$ and an effective surface of sampling of $25 \times 25 \mathrm{~cm}$. Four samples were taken in each station. The first BC was used immediately after sampling to measure temperature, $\mathrm{pH}$ and redox (Eh) at 6 and $25 \mathrm{~cm}$ below the surface of the sediment, and was subsampled later in the laboratory to analyse the sediment grain size, level of carbonates and content of organic matter. The three replica BC remaining were used to obtain the fauna. The content of each replica was sieved using three mesh sizes $(5,1$ and $0.5 \mathrm{~mm})$. The molluscan groups retained in the two largest sieves were counted to estimate their relative abundance in the total macrobenthic fauna. Afterwards, the material was fixed in formalin and preserved in $70 \%$ ethanol for further taxonomic study.

Using the superficial layer of sediment, the following percentages of granulometric fractions were recorded: coarse sand, medium sand, fine sand and mud. Carbonate content $(\%)$ was measured by treatment of the sample with hydrochloric acid, and the total organic matter content $(\mathrm{OM} \%)$ was estimated from the weight loss of the samples after $4 \mathrm{~h}$ at $450^{\circ} \mathrm{C}$ in an oven.

\section{Data analysis}

Data were organized into station by species matrices. Univariate measures were calculated for each sampling

Fig. 1 Study area and benthic sampling stations during the BENTART cruises in 2003 and 2006.

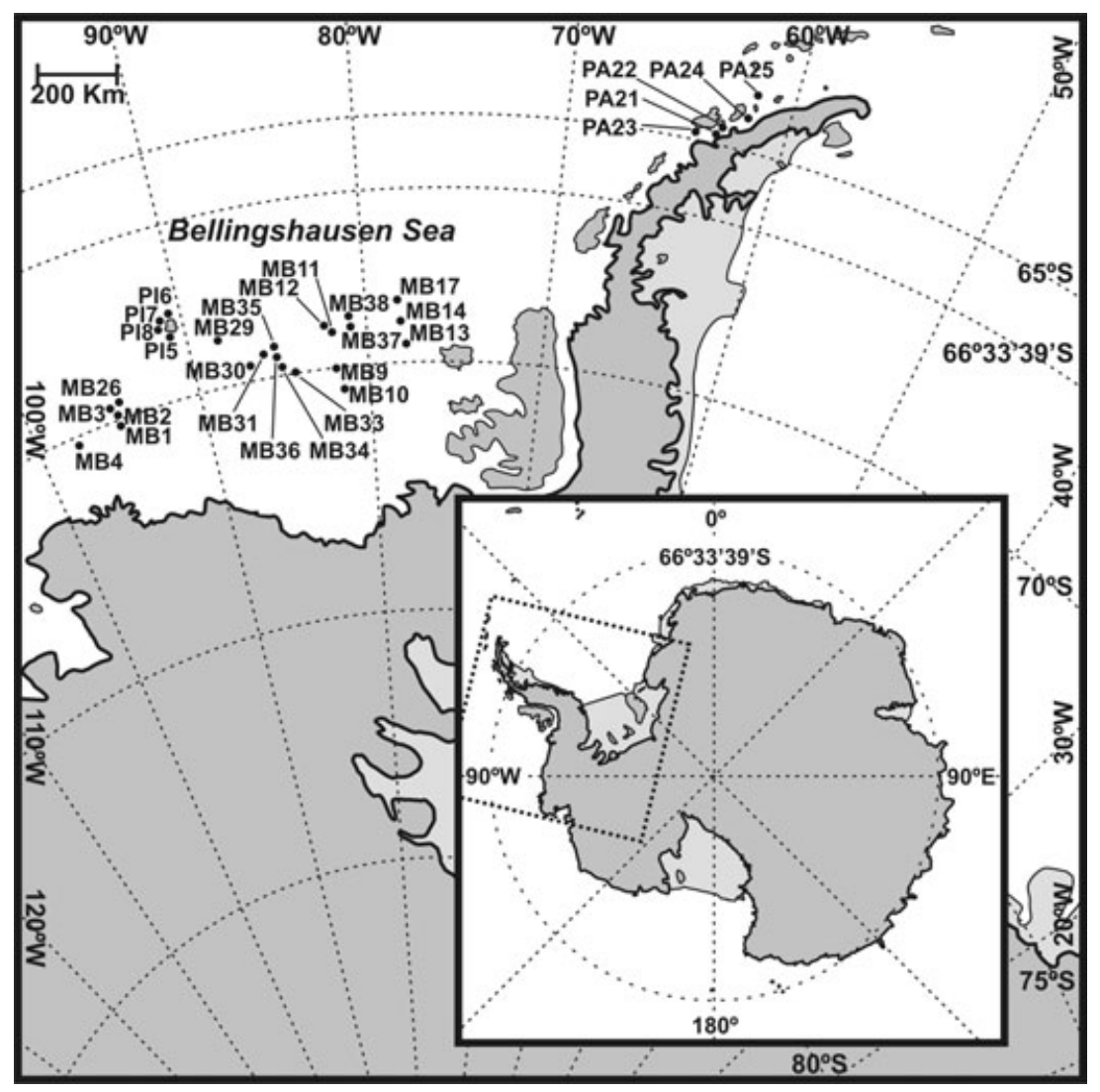


Table 1 Location, depth and environmental parameters at the surface of sediments of survey stations: redox potential (mV), organic matter (OM\%), carbonates (\%), gravel $(\%>2 \mathrm{~mm})$, coarse sand $(\%>0.5 \mathrm{~mm})$, medium sand $(\%>0.25 \mathrm{~mm})$, fine sand $(\%>0.0625 \mathrm{~mm})$ and mud $(\%<0.0625 \mathrm{~mm})$.

\begin{tabular}{|c|c|c|c|c|c|c|c|c|c|c|c|}
\hline Station & Latitude S & Longitude W & Depth (m) & Redox & $\mathrm{OM}$ & Carbonates & Gravel & Coarse sand & Medium sand & Fine sand & $\overline{\text { Mud }}$ \\
\hline MB1 (2003) & $70^{\circ} 38.22^{\prime}$ & $95^{\circ} 15.36^{\prime}$ & 534 & 252.2 & 4.81 & n.d. & 14.30 & 7.90 & 7.50 & 19.10 & 51.20 \\
\hline MB2 (2003) & $70^{\circ} 29.25^{\prime}$ & $95^{\circ} 14.83^{\prime}$ & 780 & 289.3 & 5.02 & n.d. & 81.40 & 1.80 & 1.10 & 4.20 & 11.50 \\
\hline MB3 (2003) & $70^{\circ} 17.58^{\prime}$ & $95^{\circ} 11.86^{\prime}$ & 1431 & 259.8 & 5.42 & n.d. & 29.00 & 4.30 & 3.40 & 30.50 & 32.80 \\
\hline MB4 (2003) & $70^{\circ} 52.86^{\prime}$ & $98^{\circ} 26.12^{\prime}$ & 425 & 271.3 & 4.56 & n.d. & 31.00 & 9.60 & 5.40 & 16.40 & 37.60 \\
\hline PI 5 (2003) & $68^{\circ} 56.70^{\prime}$ & $90^{\circ} 35.70^{\prime}$ & 126 & 199.3 & 1.43 & n.d. & 0.14 & 0.14 & 0.32 & 19.50 & 79.90 \\
\hline PI 6 (2003) & $68^{\circ} 49.61^{\prime}$ & $90^{\circ} 48.78^{\prime}$ & 210 & 122.5 & 1.35 & n.d. & 0.00 & 0.10 & 0.10 & 21.00 & 78.80 \\
\hline PI 7 (2003) & $68^{\circ} 42.20^{\prime}$ & $90^{\circ} 40.80^{\prime}$ & 410 & 174.8 & 1.85 & n.d. & 0.00 & 0.20 & 0.20 & 6.10 & 93.50 \\
\hline PI 8 (2003) & $68^{\circ} 50.18^{\prime}$ & $90^{\circ} 51.08^{\prime}$ & 90 & 155.8 & 1.23 & n.d. & 0.10 & 0.80 & 4.90 & 58.90 & 35.30 \\
\hline MB9 (2003) & $70^{\circ} 14.40^{\prime}$ & $81^{\circ} 47.03^{\prime}$ & 532 & 261.8 & 5.96 & n.d. & 3.90 & 6.10 & 4.40 & 12.40 & 73.20 \\
\hline MB10 (2003) & $70^{\circ} 44.31^{\prime}$ & $81^{\circ} 27.85^{\prime}$ & 497 & 260 & 4.05 & n.d. & 15.80 & 5.20 & 7.90 & 16.40 & 54.70 \\
\hline MB11 (2003) & $69^{\circ} 27.07^{\prime}$ & $82^{\circ} 06.76^{\prime}$ & 1289 & 266 & 3.81 & n.d. & 22.40 & 8.50 & 3.70 & 10.60 & 54.80 \\
\hline MB12 (2003) & $69^{\circ} 24.27^{\prime}$ & $82^{\circ} 11.88^{\prime}$ & 2032 & 261.5 & 5.29 & n.d. & 23.00 & 11.10 & 5.80 & 18.06 & 42.04 \\
\hline MB13 (2003) & $69^{\circ} 49.56^{\prime}$ & $77^{\circ} 43.68^{\prime}$ & 605 & 240.5 & 4.64 & n.d. & 10.20 & 3.60 & 4.10 & 17.50 & 64.60 \\
\hline MB14 (2003) & $69^{\circ} 21.12^{\prime}$ & $78^{\circ} 04.91^{\prime}$ & 498 & n.d. & 3.68 & n.d. & 34.70 & 5.10 & 3.80 & 11.70 & 44.70 \\
\hline MB17 (2003) & $68^{\circ} 54.88^{\prime}$ & $78^{\circ} 14.16^{\prime}$ & 2044 & 224.7 & 1.98 & n.d. & 64.50 & 18.60 & 3.90 & 2.90 & 10.10 \\
\hline PA21 (2003) & $64^{\circ} 54.01^{\prime}$ & $63^{\circ} 01.11^{\prime}$ & 107 & 133.5 & 2.49 & n.d. & 4.40 & 14.80 & 17.90 & 34.80 & 28.10 \\
\hline PA22 (2003) & $64^{\circ} 50.58^{\prime}$ & $62^{\circ} 57.91^{\prime}$ & 294 & 137 & 6.40 & n.d. & 0.00 & 0.00 & 0.30 & 3.20 & 96.50 \\
\hline PA23 (2003) & $64^{\circ} 55.95^{\prime}$ & $63^{\circ} 38.40^{\prime}$ & 655 & 272.5 & 6.75 & n.d. & 0.00 & 0.50 & 0.50 & 7.10 & 91.90 \\
\hline PA24 (2003) & $64^{\circ} 20.11^{\prime}$ & $61^{\circ} 58.82^{\prime}$ & 1056 & 170.5 & 8.32 & n.d. & 0.00 & 0.24 & 0.23 & 1.53 & 98.00 \\
\hline PA25 (2003) & $63^{\circ} 52.85^{\prime}$ & $61^{\circ} 48.52^{\prime}$ & 110 & n.d. & 1.16 & n.d. & 23.80 & 22.90 & 13.50 & 36.30 & 3.50 \\
\hline MB26 (2006) & $70^{\circ} 14.62^{\prime}$ & $95^{\circ} 02.20^{\prime}$ & 1920 & 178.9 & 1.99 & 5.87 & 1.33 & 11.22 & 29.09 & 49.43 & 8.94 \\
\hline MB29 (2006) & $69^{\circ} 26.08^{\prime}$ & $88^{\circ} 26.17^{\prime}$ & 3304 & 262.1 & 8.92 & 1.14 & 1.54 & 5.56 & 2.47 & 5.25 & 85.19 \\
\hline MB30 (2006) & $69^{\circ} 58.98^{\prime}$ & $87^{\circ} 31.08^{\prime}$ & 1814 & 187.7 & 7.01 & 2.97 & 58.38 & 1.78 & 1.02 & 8.88 & 29.95 \\
\hline MB31 (2006) & $69^{\circ} 56.98^{\prime}$ & $86^{\circ} 19.27^{\prime}$ & 1426 & 207.8 & 5.31 & 2.54 & 0.00 & 2.22 & 4.81 & 20.74 & 72.22 \\
\hline MB33 (2006) & $70^{\circ} 15.90^{\prime}$ & $84^{\circ} 11.45^{\prime}$ & 438 & 290.2 & 4.02 & 1.38 & 20.11 & 12.99 & 8.86 & 26.32 & 31.72 \\
\hline MB34 (2006) & $70^{\circ} 08.20^{\prime}$ & $84^{\circ} 51.68^{\prime}$ & 603 & 326 & 1.80 & 1.27 & 0.00 & 12.91 & 14.98 & 59.89 & 12.21 \\
\hline MB35 (2006) & $69^{\circ} 56.03^{\prime}$ & $85^{\circ} 11.30^{\prime}$ & 1117 & 260.7 & 7.36 & 2.40 & 47.65 & 3.78 & 1.73 & 9.13 & 37.72 \\
\hline MB36 (2006) & $69^{\circ} 56.28^{\prime}$ & $80^{\circ} 24.55^{\prime}$ & 560 & 289 & 8.51 & 0.47 & 33.15 & 1.08 & 1.08 & 3.96 & 60.72 \\
\hline MB37 (2006) & $69^{\circ} 26.38^{\prime}$ & $80^{\circ} 51.62^{\prime}$ & 495 & 244 & 5.70 & 0.64 & 35.37 & 17.04 & 10.27 & 16.15 & 21.17 \\
\hline MB38 (2006) & $69^{\circ} 14.08^{\prime}$ & $80^{\circ} 61.20^{\prime}$ & 1324 & 298.2 & 5.98 & 0.83 & 65.69 & 3.14 & 1.26 & 2.72 & 27.20 \\
\hline
\end{tabular}

MB, Bellingshausen Sea; n.d., not determined; PA, Antarctic Peninsula; PI, Peter I Øy. BENTART 2003, January-February 2003; BENTART 2006, January-February 2006.

station: total abundance $(N)$, number of species $(S)$, the Shannon-Wiener diversity index $\left(H^{\prime}, \log _{\mathrm{e}}\right)$ and Pielou's evenness $\left(J^{\prime}\right)$. Molluscan assemblages were determined through non-parametric multivariate techniques as described by Field et al. (1982) using the PRIMER v5.0 (Plymouth Routines in Multivariate Ecological Research) software package (Clarke \& Warwick 1994). A similarity matrix between sampling stations was constructed by means of the Bray-Curtis similarity coefficient by first applying fourth-root transformation on species abundance to downweight the contribution of the most abundant species. From this matrix, a classification of the stations was performed by cluster analysis based on the group-average sorting algorithm, as well as an ordination by means of nonmetrical multidimensional scaling (MDS). Possible differences in faunistic composition between the two major groups of stations were tested using a one-way ANOSIM test. The SIMPER program was then used to identify species that greatly contributed to the differentiation of station groups.
The BIO-ENV procedure was used to research the possible relationship between molluscan distributions in the Bellingshausen Sea and the measured environmental variables. The following variables were considered in this analysis: depth (m), redox (Eh), OM (\%) and all granulometric fractions (\%). Carbonates were discarded from the analysis because of the lack of such data from BENTART 03. All variables were previously transformed by $\log (x+1)$.

\section{Results}

\section{Abundance, number of species and diversity}

A total of 1074 molluscan specimens were collected belonging to 62 species in four classes. The bestrepresented class, in terms of number of species, was Gastropoda (14 families and 33 species), followed by Bivalvia (15 families and 25 species), Scaphopoda (three families and three species) and Polyplacophora (one 
family and one species) (Table 2, Fig. 2). The bivalves Thyasira bongraini and Cyamiocardium denticulatum accounted for $60 \%$ of all molluscs.

The number of molluscs species per sampling site ranged from 1 to 14 (Table 3). Maximal abundances were found at sites PI5 (446) and PI8 (244), and the lowest were found at sites MBI, MB10, MB12 and MB17, with only one specimen in each. In MB26, MB29 and MB34, no molluscs species were obtained. The highest diversity was recorded at site PA2 $1\left(H^{\prime}=2.36\right)$ in contrast to that found at MB9 $\left(H^{\prime}=0.27\right)$. $J^{\prime}$ is usually high in sampling sites, except in stations near Peter I Øy.
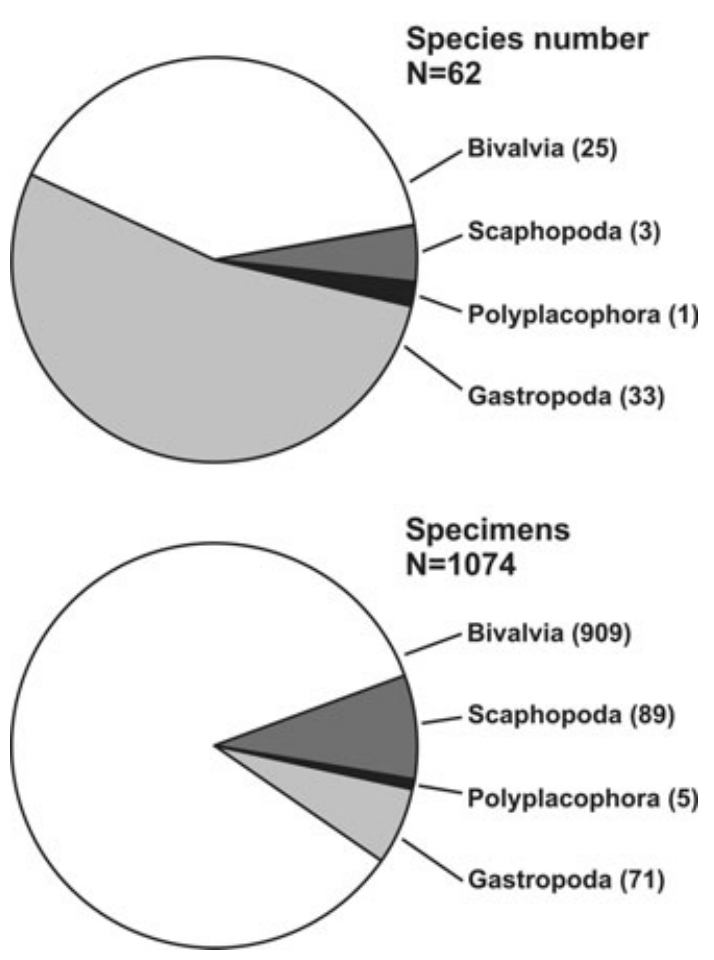

Fig. 2 Number of species and specimens of each molluscan class.

\section{Molluscan assemblages}

The cluster analysis (Fig. 3) showed the presence of major groups of sites at a similarity level of $20 \%$ : group A (six shallow-water stations, 90-410 $\mathrm{m}$ in depth, high $\%$ of mud and low values of OM) and group B (16 deepwater stations, 438-2044 $\mathrm{m}$ in depth, medium values of mud \% and high OM). MDS ordination (Fig. 4) showed similar results to those of the dendrogram, with an acceptable stress value (0.05). Two major groups (A and B) are segregated from right to left, which can be identified as the depth gradient from shallow stations off Peter I $\varnothing y$ and the Antarctic Peninsula to deeper stations in the Bellingshausen Sea. The ANOsIm test showed significant differences on the faunistic composition between groups A and B according to the depth factor: shallow (group A) vs. deep (group B) stations (global $R=0.571$, $p=0.001)$.

Results of the SIMPER analysis for the dissimilarity between groups are shown in Table 4 . The bivalves Thyasira bongraini, Cyamiocardium denticulatum, Cuspidaria infelix, Adacnarca nitens, Cyclocardia astartoides, Limopsis lilliei and the scaphopod Siphonodentalium dalli f. antarcticus contributed greatly to the similarity (up to a cumulative $92 \%$ ) in populations found for shallow-water stations of group A. The deeper-water of group B is mainly determined by Dentalium majorinum. The species that contribute most to the dissimilarity between the two groups according to ratio values were $T$. bongraini, D. majorinum, C. infelix and C. denticulatum.

\section{Relationship between biotic and environmental variables}

Sediments were predominantly mud, with relatively high contents of organic matter (Table 1). The superficial sediments appeared to be oxidized, as shown by redox (Eh) values of greater than $122.5 \mathrm{mV}$. Carbonate content was low in stations of BENTART 06 (MB26, 29, 30, 31, 33, 34,

Table 2 Systematic list of species found, indicating stations where these were collected.

\begin{tabular}{lll}
\hline Class/Family & Species & Station \\
\hline $\begin{array}{l}\text { POLYPLACOPHORA } \\
\text { Leptochitonidae }\end{array}$ & Leptochiton kerguelenensis Haddon, 1886 & MB11, MB14, MB17, PA23 \\
GASTROPODA & & MB31 \\
Anatomidae & Anatoma euglypta (Pelseneer, 1903) & MB37 MB4 \\
Trochidae & Antimargarita sp. & MB30 \\
& Calliotropis pelseneeri Cernohorsky, 1977 & PI8 \\
& Calliotropis sp. & MB11
\end{tabular}


Table 2 Continued

\begin{tabular}{|c|c|c|}
\hline Class/Family & Species & Station \\
\hline Zerotulidae & Dickdellia labioflecta (Dell, 1990) & PA22 \\
\hline Eatoniellidae & Eatoniella glacialis (Smith, 1907) & PA21 \\
\hline \multirow[t]{2}{*}{ Rissoidae } & Onoba gelida (Smith, 1907) & $\mathrm{PI} 8, \mathrm{PA} 21$ \\
\hline & Onoba kergueleni (Smith, 1875) & $\mathrm{PI} 8$ \\
\hline Capulidae & Torellia planispira (Smith, 1915) & PA21 \\
\hline \multirow[t]{3}{*}{ Naticidae } & Amauropsis anderssoni (Strebel, 1906) & PA25 \\
\hline & Amauropsis aureolutea (Strebel, 1908) & MB11 \\
\hline & Falsilunatia delicatula (Smith, 1902) & $\mathrm{Pl} 5, \mathrm{Pl} 8$ \\
\hline Eulimidae & Balcis antarctica (Strebel, 1908) & MB13 \\
\hline \multirow[t]{3}{*}{ Muricidae } & Trophon cuspidarioides Powell, 1951 & $\mathrm{PI} 7$ \\
\hline & Trophon drygalskii Thiele, 1912 & MB31 \\
\hline & Trophon longstaffi Smith, 1907 & $\mathrm{Pl} 5$ \\
\hline \multirow[t]{6}{*}{ Buccinidae } & Chlanidota signeyana Powell, 1951 & $\mathrm{PI5}, \mathrm{PI} 7, \mathrm{PA} 24$ \\
\hline & Neobuccinum eatoni (Smith, 1875) & $\mathrm{PI5}$ \\
\hline & Pareuthria regulus (Watson, 1882) & PI5 \\
\hline & Probuccinum costatum Thiele, 1912 & $\mathrm{PI} 7$ \\
\hline & Prosipho chordatus (Strebel, 1908) & $\mathrm{PI} 8$ \\
\hline & Prosipho hedleyi Powell, 1958 & $\mathrm{Pl} 8$ \\
\hline Volutidae & Harpovoluta charcoti (Lamy, 1910) & PA23 \\
\hline Conidae & Belaturricula gaini (Lamy, 1910) & PA23 \\
\hline \multirow[t]{4}{*}{ Turridae } & Leucosyrinx paratenoceras Powell, 1951 & MB36 \\
\hline & Lorabela sp. & $\mathrm{Pl} 5, \mathrm{Pl} 8$ \\
\hline & Pleurotomella simillina Thiele, 1912 & $\mathrm{PI7}$ \\
\hline & Typhlodaphne innocentia Dell, 1990 & $\mathrm{Pl} 5, \mathrm{Pl} 8$ \\
\hline \multirow{2}{*}{ Acteonidae } & Acteon antarcticus Thiele, 1912 & MB3 \\
\hline & Neactaeonina cf. edentula (Watson, 1883) & PI5 \\
\hline \multicolumn{3}{|l|}{ BIVALVIA } \\
\hline Nuculanidae & Propeleda longicaudata (Thiele, 1912) & PA21, MB33 \\
\hline \multirow[t]{5}{*}{ Yoldiidae } & Yoldia eightsi (Couthouy, 1839) & PA22 \\
\hline & Yoldiella antarctica (Thiele, 1912) & $\mathrm{PI7}$ \\
\hline & Yoldiella ecaudata (Pelseneer, 1903) & MB3, MB36 \\
\hline & Yoldiella oblonga (Pelseneer, 1903) & MB9, MB14 \\
\hline & Yoldiella profundorum (Melvill \& Standen, 1912) & PA22 \\
\hline Malletiidae & Malletia pellucida Thiele, 1912 & MB31, MB35 \\
\hline Arcidae & Bathyarca sinuata Pelseneer, 1903 & MB3, MB13, MB30, MB35, MB38 \\
\hline \multirow[t]{2}{*}{ Limopsidae } & Limopsis lilliei Smith, 1915 & PA21, PA25 \\
\hline & Limopsis longipilosa Pelseneer, 1903 & MB33, MB37 \\
\hline \multirow[t]{2}{*}{ Philobryidae } & Philobrya sublaevis (Pelseneer, 1903) & $\mathrm{Pl} 8$ \\
\hline & Adacnarca nitens Pelseneer, 1903 & $\mathrm{PI5}, \mathrm{PI} 8, \mathrm{PA} 21$ \\
\hline Mytilidae & Dacrydium albidum Pelseneer, 1903 & MB38 \\
\hline Limidae & Limatula simillina Thiele, 1912 & MB37 \\
\hline Pectinidae & Adamussium colbecki (Smith, 1902) & MB2, MB3, PI5, PI7, MB30, MB31, MB35 \\
\hline \multirow[t]{2}{*}{ Thyasiridae } & Thyasira bongraini (Lamy, 1910) & $\mathrm{PI} 5, \mathrm{Pl} 6, \mathrm{PI}, \mathrm{PI} 8, \mathrm{PA} 21, \mathrm{PA} 22, \mathrm{PA} 25$ \\
\hline & Thyasira dearborni Nicol, 1965 & PA22 \\
\hline \multirow[t]{2}{*}{ Lasaeidae } & Mysella antarctica (Smith, 1907) & PA22 \\
\hline & Pseudokellya cardiformis (Smith, 1885) & PA21 \\
\hline \multirow[t]{2}{*}{ Cyamiidae } & Cyamiocardium denticulatum (Smith, 1907) & MB4, PI5, PI7, PI8, PA21 \\
\hline & Cyamiomactra laminifera $($ Lamy, 1906) & $\mathrm{Pl} 8$ \\
\hline \multirow[t]{2}{*}{ Carditidae } & Cyclocardia astartoides (Martens, 1878) & PA21, PA25 \\
\hline & Cyclocardia cf. intermedia Thiele, 1912 & MB4 \\
\hline Thraciidae & Thracia meridionalis Smith, 1885 & PA21 \\
\hline Cuspidariidae & Cuspidaria infelix Thiele, 1912 & $\mathrm{PI} 5, \mathrm{PI} 7, \mathrm{PI} 8, \mathrm{PA} 21$ \\
\hline \multicolumn{3}{|l|}{ SCAPHOPODA } \\
\hline Dentaliidae & Dentalium majorinum Mabille \& Rochebrune, 1891 & $\begin{array}{l}\text { MB1, MB2, PI7, MB9, MB10, MB13, MB14, MB31, } \\
\text { MB33, MB35, MB36, MB38 }\end{array}$ \\
\hline Rhabdidae & Rhabdus cf. perceptus (Mabille \& Rochebrune, 1891) & $\mathrm{PI}, \mathrm{MB} 12$ \\
\hline Gadilidae & Siphonodentalium dalli f. antarcticus (Odhner, 1931) & $\mathrm{PI} 5, \mathrm{Pl} 6, \mathrm{PI} 7, \mathrm{PA} 24$ \\
\hline
\end{tabular}


35, 36, 37, 38 and 39). The depths in the sampling sites of the Bellingshausen Sea varied from 90 to 3304 metres. The ANOSIm test also showed differences in faunistic composition among stations following a gradient of depth.

Table 3 Faunistic parameters at each station: species richness $(R)$, total abundante $(N)$, Pielou even ness index $\left(J^{\prime}\right)$ and Shannon-Wiener diversity index calculated with the natural logarithm $\left(H^{\prime}\right)$, MO data (***).

\begin{tabular}{|c|c|c|c|c|}
\hline Station & Richness (S) & Abundance $(N)$ & Evenness $\left(J^{\prime}\right)$ & Diversity $\left(H^{\prime}\right)$ \\
\hline MB1 & 1 & 1 & $\star * *$ & 0 \\
\hline MB2 & 2 & 2 & 1 & 0.69 \\
\hline MB3 & 4 & 5 & 0.96 & 1.33 \\
\hline MB4 & 3 & 3 & 1 & 1.10 \\
\hline PI5 & 14 & 446 & 0.40 & 1.06 \\
\hline Pl6 & 2 & 145 & 0.72 & 0.50 \\
\hline $\mathrm{PI7}$ & 12 & 67 & 0.58 & 1.45 \\
\hline PI8 & 14 & 244 & 0.49 & 1.29 \\
\hline MB9 & 2 & 13 & 0.39 & 0.27 \\
\hline MB10 & 1 & 1 & $\star \star \star *$ & 0 \\
\hline MB11 & 3 & 3 & 1 & 1.10 \\
\hline MB12 & 1 & 2 & $\star \star \star *$ & 0 \\
\hline MB13 & 3 & 3 & 1 & 1.10 \\
\hline MB14 & 3 & 7 & 0.87 & 0.96 \\
\hline MB17 & 1 & 1 & $\star * *$ & 0 \\
\hline PA21 & 13 & 22 & 0.92 & 2.36 \\
\hline PA22 & 6 & 55 & 0.91 & 1.63 \\
\hline PA23 & 3 & 3 & 1 & 1.10 \\
\hline PA24 & 2 & 2 & 1 & 0.69 \\
\hline PA25 & 4 & 7 & 0.92 & 1.28 \\
\hline MB30 & 3 & 8 & 0.82 & 0.90 \\
\hline MB31 & 5 & 7 & 0.96 & 1.55 \\
\hline MB33 & 3 & 3 & 1 & 1.10 \\
\hline MB35 & 4 & 4 & 1 & 1.39 \\
\hline MB36 & 3 & 7 & 0.72 & 0.80 \\
\hline MB37 & 3 & 7 & 0.72 & 0.80 \\
\hline MB38 & 3 & 6 & 0.92 & 1.01 \\
\hline
\end{tabular}

The BIO-ENV procedure (Table 5) showed that the combination of environmental variables with the highest correlations with faunistic data was depth and coarse sand at the surface. Redox (Eh) and fine sand at the surface were not involved in the best combinations. Depth was the variable with the best value when each variable was considered alone $\left(p_{\mathrm{w}}=0.390\right)$.

\section{Discussion}

Linse et al. (2006) divided the Southern Ocean into seven subregions based on richness hotspots of gastropods and bivalves: Antarctic Peninsula, Weddell Sea, three East Antarctic subregions (Dronning Maud Land, Enderby Land and Wilkes Land), Ross Sea, and the independent Scotia Arc and sub-Antarctic islands, and excluded the West Antarctic Region (Eights Coast and the Bellingshausen and Amundsen seas), previously defined by Clarke et al. (2004) from this classification because it is insufficiently studied. So, the present study, together with future more comprehensive faunistic studies (Aldea $\delta$ Troncoso, unpubl. data), represents notable progress in the knowledge of the Bellingshausen Sea.

For neighbouring areas, several surveys of semiquantitative (e.g. Arnaud et al. 1998) and quantitative analyses of molluscan or macrobenthic fauna of the soft bottom have appeared in recent literature (e.g. Sáiz-Salinas et al. 1997; Sahade et al. 1998; Arnaud et al. 2001) showing distribution patterns and assemblages of several species, mainly from north-west of the Antarctic Peninsula. Likewise, similar studies about the Ross Sea have previously been published (e.g. Russo \& Gambi 1994; Gambi et al. 1997). Only one survey of the benthic marine fauna of
Fig. 3 Faunistic assemblages in the study are determined by cluster analysis based on BrayCurtis Similarity.

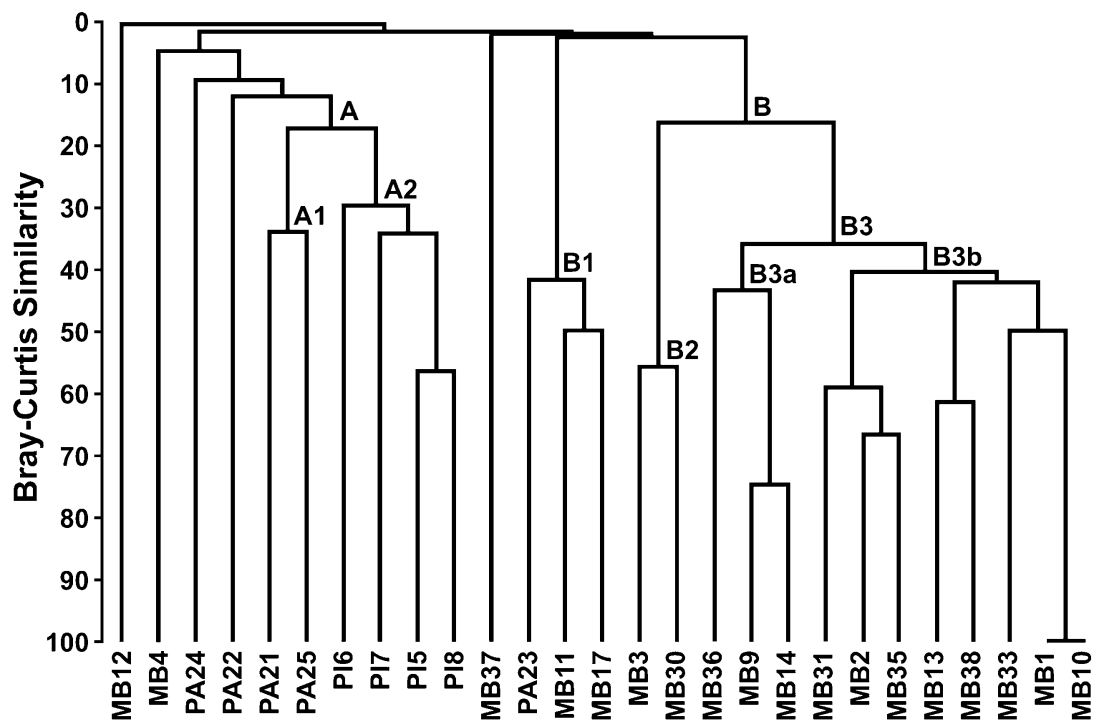




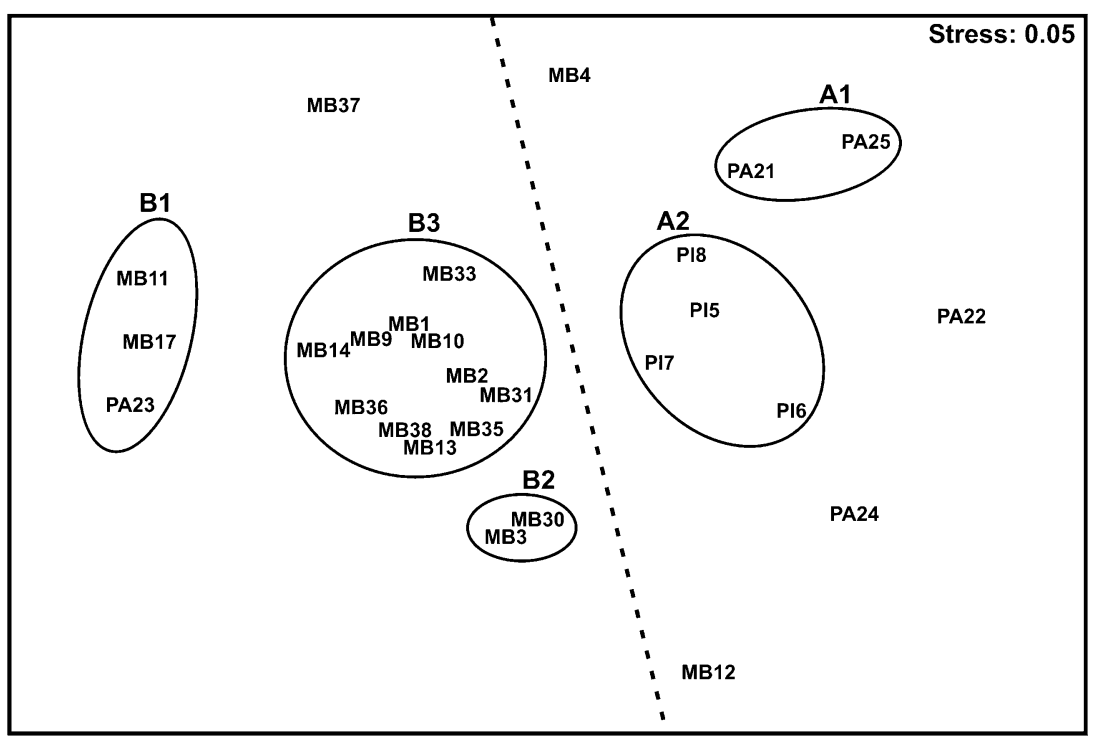

Fig. 4 MDS ordination of faunistic assemblages. The dotted line represents the separation of two main groups (A, B). The subgroups delimited with solid lines are derivates of cluster analysis.

Table 4 Results of SIMPER analysis. Species were ranked according to their average contribution to dissimilarity (Contrib. \%) between groups of stations. Average abundance for each group (Av. abund.), average dissimilarity (Av. diss.), ratio value (dissimilarity/standard deviation, Diss./SD) and percentage of cumulative dissimilarity (Cum. \%).

\begin{tabular}{|c|c|c|c|c|c|c|}
\hline Groups B \& A (Average dissimilarity = 96.79) & Group B & Group A & & & & \\
\hline Species & Av. abund. & Av. abund. & Av. diss. & Diss./SD & Contrib. \% & Cum. \% \\
\hline Thyasira bongraini & 0 & 2.36 & 16.46 & 1.54 & 17.01 & 17.01 \\
\hline Dentalium majorinum & 1.21 & 0.31 & 8.47 & 1.54 & 8.75 & 25.76 \\
\hline Cyamiocardium denticulatum & 0 & 1.64 & 7.17 & 1.12 & 7.41 & 33.17 \\
\hline Siphonodentalium dalli f. antarcticus & 0 & 0.86 & 6.72 & 0.67 & 6.94 & 40.11 \\
\hline Cuspidaria infelix & 0 & 1.03 & 4.74 & 1.36 & 4.9 & 45.01 \\
\hline Cyclocardia astartoides & 0 & 0.4 & 3.87 & 0.62 & 4 & 49.01 \\
\hline Limopsis lilliei & 0 & 0.33 & 3.26 & 0.62 & 3.37 & 52.38 \\
\hline Amauropsis anderssoni & 0 & 0.22 & 3.01 & 0.44 & 3.11 & 55.49 \\
\hline Adacnarca nitens & 0 & 0.65 & 2.9 & 0.98 & 2.99 & 58.48 \\
\hline Adamussium colbecki & 0.29 & 0.33 & 2.76 & 0.72 & 2.85 & 61.33 \\
\hline Bathyarca sinuata & 0.29 & 0 & 2.04 & 0.53 & 2.11 & 63.44 \\
\hline Onoba gelida & 0 & 0.39 & 1.86 & 0.7 & 1.92 & 65.37 \\
\hline Chlanidota signeyana & 0 & 0.36 & 1.75 & 0.67 & 1.81 & 67.18 \\
\hline Falsilunatia delicatula & 0 & 0.4 & 1.62 & 0.68 & 1.67 & 68.85 \\
\hline Lorabela sp. & 0 & 0.39 & 1.55 & 0.69 & 1.6 & 70.45 \\
\hline Typhlodaphne innocentia & 0 & 0.36 & 1.47 & 0.7 & 1.51 & 71.97 \\
\hline Propeleda longicaudata & 0.09 & 0.17 & 1.46 & 0.49 & 1.51 & 73.48 \\
\hline Yoldiella oblonga & 0.18 & 0 & 1.31 & 0.41 & 1.36 & 74.83 \\
\hline Torellia planispira & 0 & 0.22 & 1.27 & 0.44 & 1.32 & 76.15 \\
\hline Thracia meridionalis & 0 & 0.22 & 1.27 & 0.44 & 1.32 & 77.47 \\
\hline Pleurotomella simillina & 0 & 0.22 & 1.21 & 0.44 & 1.25 & 78.71 \\
\hline Malletia pellucida & 0.18 & 0 & 1.17 & 0.42 & 1.2 & 79.92 \\
\hline Neactaeonina cf. edentula & 0 & 0.26 & 1.03 & 0.44 & 1.07 & 80.99 \\
\hline Submargarita notalis & 0 & 0.17 & 0.97 & 0.44 & 1 & 81.99 \\
\hline Eatoniella glacialis & 0 & 0.17 & 0.97 & 0.44 & 1 & 82.99 \\
\hline Pseudokellya cardiformis & 0 & 0.17 & 0.97 & 0.44 & 1 & 83.99 \\
\hline Philobrya sublaevis & 0 & 0.24 & 0.96 & 0.44 & 0.99 & 84.98 \\
\hline Trophon cuspidarioides & 0 & 0.17 & 0.92 & 0.44 & 0.95 & 85.92 \\
\hline Probuccinum costatum & 0 & 0.17 & 0.92 & 0.44 & 0.95 & 86.87 \\
\hline Yoldiella antarctica & 0 & 0.17 & 0.92 & 0.44 & 0.95 & 87.82 \\
\hline Rhabdus cf. perceptus & 0 & 0.17 & 0.92 & 0.44 & 0.95 & 88.77 \\
\hline Margarella antarctica & 0 & 0.2 & 0.81 & 0.44 & 0.83 & 89.6 \\
\hline Prosipho hedleyi & 0 & 0.2 & 0.81 & 0.44 & 0.83 & 90.44 \\
\hline
\end{tabular}


Table 5 Best combinations of variables obtained through BIO-ENV analysis according to the values of the Spearman's rank correlation $\left(p_{w}\right)$ for survey stations: depth $(\mathrm{m})$, organic matter (OM \%), gravel $(\%>2 \mathrm{~mm})$, coarses and $(\%>0.5 \mathrm{~mm})$, mediums and $(\%>0.25 \mathrm{~mm})$ and mud $(\%<0.0625 \mathrm{~mm})$.

\begin{tabular}{|c|c|c|}
\hline $\begin{array}{l}\text { Number of } \\
\text { variables }\end{array}$ & $\begin{array}{l}\text { Correlation } \\
\left(p_{\mathrm{w}}\right)\end{array}$ & Best variable combination \\
\hline 2 & 0.454 & depth; coarse sand \\
\hline 3 & 0.410 & depth; OM; coarse sand \\
\hline 1 & 0.390 & Depth \\
\hline 3 & 0.385 & depth; gravel; coarse sand \\
\hline 3 & 0.382 & depth; coarse sand; mud \\
\hline 4 & 0.378 & depth; OM; coarse sand \\
\hline 3 & 0.378 & depth; coarse sand; medium sand \\
\hline 4 & 0.368 & depth; OM; coarse sand; medium sand \\
\hline 4 & 0.362 & depth; OM; coarse sand; mud \\
\hline 3 & 0.359 & depth; OM; gravel \\
\hline
\end{tabular}

the Bellingshausen Sea has been published previously (Richardson \& Hedgpeth 1977).

The main species recorded in the present survey are the same as those recorded during a similar survey in the South Shetland Islands and Bransfield Strait (Arnaud et al. 2001): the tiny Cyamiocardium denticulatum and Thyasira bongraini, and the large Dentalium majorinum, Siphonodentalium dalli f. antarcticus, Cuspidaria infelix and Yoldia eightsi. Mühlenhardt-Siegel (1989) also found a high presence of T. bongraini. The same situation occurs in the Ross Sea, where Cattaneo-Vietti et al. (2000) found mainly the bivalves Adamussium colbecki, Galeommatidae undet., Y. eightsi and T. dearborni (named as Genaxinus debilis).

Molluscan assemblages exhibit a bathymetric pattern from deep-water stations (depth $>438 \mathrm{~m}$ ) to shallowwater ones (depth $<410 \mathrm{~m})$. Deep-water stations with very low abundances $(<13$ specimens) mainly belong to group B, which is widely dominated by the large $D$. majorinum, followed by the bivalves $A$. colbeki, Bathyarca sinuata and the tiny species Limopsis longipilosa and the chiton Leptochiton kerguelenensis. On the contrary, shallow-water stations with high abundances (22-446 specimens), belonging mainly to group A, and mostly located near the Antarctic Peninsula or Peter I Øy, are dominated by the tiny $C$. denticulatum and T. bongraini, followed by the larger scaphopod S. dalli f. antarcticus, C. infelix and Y. eightsi. This result agrees with those of studies carried out in adjacent areas at depths generally shallower than 500 m, where Arnaud et al. (2001) have reported the high presence and abundances of the bivalves $C$. denticulatum and T. bongraini. However, this result disagrees with the survey of Cattaneo-Vietti et al. (2000), who found mainly large bivalves (A. colbecki, $Y$. eightsi) in depths of less than $380 \mathrm{~m}$, and the tiny infaunal bivalve T. dearborni (under the name G. debilis) at depths of $222-1000 \mathrm{~m}$. This is an inverse pattern, with tiny infaunal molluscs dominating at greater depths.

In conclusion, the main molluscan species of the Ross Sea (Schiaparelli et al. 2006), Weddell Sea (Hain 1990) and the South Shetland Islands (Arnaud et al. 2001) are also present in the Bellingshausen Sea, but in lesser abundance. Peter I Øy presents the highest values of abundances and number of species. T. bongraini and C. denticulatum contribute $60 \%$ of the total number of the specimens. Depth and percentage of coarse sand could be the environmental variables that explain the distribution of molluscan assemblages in the Bellingshausen Sea.

\section{Acknowledgements}

This research has been supported by the Spanish Government through the Ministry of Education and Science. The officers and crew of the RV Hesperides and our colleagues from the BENTART cruises in 2003 and 2006 played a prominent part in the success of this project, and we express our gratitude to all of them. We are also grateful to Juan Moreira and two anonymous referees for their helpful comments that improved the manuscript.

\section{References}

Arnaud P. M., López C.M., Olaso I., Ramil F., Ramos-Esplá A.A. \& Ramos A. 1998. Semi-quantitative study of macrobenthic fauna in the region of the South Shetland Islands and the Antarctic Peninsula. Polar Biology 19, 160-166.

Arnaud P. M., Troncoso J. S. \& Ramos A. 2001. Species diversity and assemblages of macrobenthic Mollusca from the South Shetland Islands and Bransfield Strait (Antarctica). Polar Biology 24, 105-112.

Cattaneo-Vietti R., Chiantore M., Schiaparelli S. \& Albertelli G. 2000. Shallow- and deep-water mollusc distribution at Terra Nova Bay (Ross Sea, Antarctica). Polar Biology 23, 173-182.

Clarke A., Aronson R.B., Crame J.A., Gili J.M. \& Blake D.B. 2004. Evolution and diversity of the benthic fauna of the Southern Ocean continental shelf. Antarctic Science 16, 559568.

Clarke K.R. \& Warwick, R.M. 1994. Changes in marine communities: an approach to statistical analysis and interpretation. Plymouth: Natural Environmental Research Council, Plymouth Marine Laboratory.

Field J. G., Clarke K.R. \& Warwick, R.M. 1982. A practical strategy for analysing multispecies distributions patterns. Marine Ecology Progress Series 8, 37-52.

Gambi M.C., Castelli A. \& Guizzardi M. 1997. Polychaete populations of the shallow soft bottoms off Terra Nova Bay (Ross Sea, Antarctica): distribution, diversity and biomass. Polar Biology 17, 199-210.

Hain S. 1990. Die beschalten Mollusken (Gastropoda und Bivalvia) des Weddellmeeres, Antarktis. (The shell-bearing 
Mollusca [Gastropoda and Bivalvia], Weddell Sea, Antarctica.) Berichte zur Polarforschung 70, 1-181.

Linse K., Griffiths H.J., Barnes D.K.A. \& Clarke A. 2006. Biodiversity and biogeography of Antarctic and sub-Antarctic mollusca. Deep-Sea Research II 53, 985-1008.

Mühlenhardt-Siegel U. 1989. Quantitative investigations of Antarctic zoobenthos communities in winter (May/June) 1986 with special reference to the sediment structure. Archiv für Fischereiwissenschaft 39, 123-141.

Richardson M.D. \& Hedgpeth J.W. 1977. Antarctic soft bottom, macrobenthic community adaptations to a cold, stable, highly productive glacially affected environment. In G.A. Llano (ed.): Adaptations within Antarctic ecosystems. Washington DC: The Smithonian Institution.

Russo G.F. \& Gambi M.C. 1994. First quantitative data on coastal soft bottoms populations off Terra Nova Bay (Ross
Sea, Antarctica): bivalve molluscs. National Scientific Committee, Antarctic Oceanographic Campaign 1989-90, Data Report (1994) II. Genoa: Ministry of University, Scientific and Technological Research.

Sahade R.J., Tatian M., Kowalke J., Kühne S. \& Esnal G.B. 1998. Benthic faunal associations on soft substrates at Potter Cove, King George Island, Antarctica. Polar Biology 19, 85-91.

Sáiz-Salinas J.I., Ramos A., García F.J., Troncoso J.S., San Martin G., Sanz C. \& Palacin C. 1997. Quantitative analysis of macrobenthic soft-bottom assemblages in South Shetland waters (Antarctica). Polar Biology 17, 393-400.

Schiaparelli S., Lörz A. \& Cattaneo-Vietti R. 2006. Diversity and distribution of mollusc assemblages on the Victoria Land coast and the Balleny Islands, Ross Sea, Antarctica. Antarctic Science 18, 615-631. 